\title{
Remdesivir for the treatment of COVID-19: A living systematic review protocol
}

AUTHORS

Francisca Verdugo

0000-0003-0199-9744

Epistemonikos Foundation

UC Evidence Center, Cochrane Chile Associated Center, Pontificia Universidad Católica de Chile, Santiago, Chile

María Paz Acuña

0000-0001-7003-495X

Unidad de Infectología, Hospital Dr Sótero del Río, Santiago, Chile

Unidad de Infectología, Hospital Clínico Dra Eloísa Díaz, La Florida, Santiago, Chile

\section{Iván Solá}

0000-0003-0078-3706

Biomedical Research Institute Sant Pau, Barcelona Spain

Iberoamerican Cochrane Centre, Barcelona Spain

CIBER Epidemiología y Salud Pública (CIBERESP)

\section{Gabriel Rada}

0000-0003-2435-0710

Epistemonikos Foundation

UC Evidence Center, Cochrane Chile Associated Center, Pontificia Universidad Católica de Chile, Santiago, Chile

\section{Corresponding author:}

\section{Francisca Verdugo}

Email address: fverdugo@epistemonikos.org

Postal address: Holanda 895, Providencia, Santiago, Chile. 


\section{ABSTRACT \\ Objective}

This living systematic review aims to provide a timely, rigorous and continuously updated summary of the evidence available on the role of remdesivir in the treatment of patients with COVID-19.

\section{Data sources}

We will conduct searches in the L.OVE (Living OVerview of Evidence) platform for COVID-19, a system that maps PICO questions to a repository maintained through regular searches in electronic databases, preprint servers, trial registries and other resources relevant to COVID-19. No date or language restrictions will be applied.

\section{Eligibility criteria for selecting studies and methods}

We adapted an already published common protocol for multiple parallel systematic reviews to the specificities of this review.

We will include randomised trials evaluating the effect of remdesivir, as monotherapy or in combination with other drugs, versus placebo or no treatment in patients with COVID-19.

Two reviewers will independently screen each study for eligibility, extract data, and assess the risk of bias. We will pool the results using meta-analysis and will apply the GRADE system to assess the certainty of the evidence for each outcome.

A living, web-based version of this review will be openly available during the COVID-19 pandemic. We will resubmit it every time the conclusions change or whenever there are substantial updates.

\section{Ethics and dissemination}

No ethics approval is considered necessary. The results of this review will be widely disseminated via peer-reviewed publications, social networks and traditional media.

\section{PROSPERO Registration}

CRD42020183384

\section{Keywords}

COVID-19, severe acute respiratory syndrome coronavirus 2, Coronavirus Infections, Systematic review, Remdesivir, Antivirals 


\section{INTRODUCTION}

COVID-19 is an infection caused by the SARS-CoV-2 coronavirus [1]. It was first identified in Wuhan, China, on December 31, 2019 [2]; five months later, more than ten million cases of contagion had been identified across 188 countries [3]. On March 11, 2020, WHO characterised the COVID-19 outbreak as a pandemic [1].

While the majority of cases result in mild symptoms, some might progress to pneumonia, acute respiratory distress syndrome and death $[4],[5],[6]$. The case fatality rate reported across countries, settings and age groups is highly variable, but it ranges from about $0.5 \%$ to $10 \%$ [7]. In hospitalised patients, it has been reported to be higher than $10 \%$ in some centres [8].

One of the strategies underway to identify effective interventions for COVID-19 is repurposing drugs that have been used for the treatment of other diseases.

Remdesivir is among these investigational medications. It is a directly acting antiviral agent, initially developed for the treatment of Ebola virus during the 2014 outbreak in Western Africa [9].

Remdesivir displays antiviral activity against many RNA viruses including SARS-CoV-2, in both in vitro [10] and animal studies [11].

Following the publication of the ACTT-1, a trial conducted by the National Institute of Allergy and Infectious Diseases (NIAID), the US Food and Drug Administration issued an emergency use authorisation of remdesivir for the treatment of COVID-19 [12].

However, the results of ACTT-1 were questioned immediately, particularly for the decision to stop it early for benefit [13]. On the other hand, the decision of the government of the United States of buying virtually all stocks of the drug, generated an urgent need of independent, transparent information about the effects of remdesivir for COVID-19.

Using innovative and agile processes, taking advantage of technological tools, and resorting to the collective effort of several research groups, this living systematic review aims to provide a timely, rigorous and continuously updated summary of the evidence available on the effects of remdesivir in patients with COVID-19 . 


\section{METHODS}

This manuscript complies with the 'Preferred Reporting Items for Systematic reviews and Meta-Analyses' (PRISMA-P) guidelines for reporting protocols of systematic reviews and meta-analyses [14].

A protocol stating the shared objectives and methodology of multiple evidence syntheses (systematic reviews and overviews of systematic reviews) to be conducted in parallel for different questions relevant to COVID-19 was published elsewhere [15]. The review was registered in PROSPERO with the number CRD42020183384.

\section{Search strategies}

\section{Electronic searches}

Our literature search was devised by the team maintaining the L.OVE platform (https://app.iloveevidence.com), using the following approach:

Identification of terms relevant to the population and intervention components of the search strategy, using Word2vec technology [16] to the corpus of documents available in Epistemonikos Database.

Discussion of terms with content and methods experts to identify relevant, irrelevant and missing terms.

Creation of a sensitive boolean strategy encompassing all the relevant terms

Iterative analysis of articles missed by the boolean strategy, and refinement of the strategy accordingly.

Our main search source will be a repository developed and maintained by Epistemonikos Foundation through the screening of different sources relevant to COVID-19. At the time of submitting this protocol, this repository includes more than 62000 articles, with 93655 directly relevant to Coronavirus disease, coming from the following databases, trial registries, preprint servers, repositories and websites relevant to COVID-19: Epistemonikos database, Pubmed, EMBASE, ICTRP Search Portal, Clinicaltrials.gov, ISRCTN registry, Chinese Clinical Trial Registry, IRCT - Iranian Registry of Clinical Trials, EU Clinical Trials Register: Clinical trials for covid-19, NIPH Clinical Trials Search (Japan) - Japan Primary Registries Network (JPRN) (JapicCTI, JMACCT CTR, jRCT, UMIN CTR), UMIN-CTR - UMIN Clinical Trials Registry, JRCT Japan Registry of Clinical Trials, JAPIC Clinical Trials Information, Clinical Research Information Service (CRiS), Republic of Korea, ANZCTR - Australian New Zealand Clinical Trials Registry, ReBec - Brazilian Clinical Trials Registry, CTRI - Clinical Trials Registry - India, DRKS German Clinical Trials Register, LBCTR - Lebanese Clinical Trials Registry, TCTR - Thai Clinical Trials Registry, NTR - The Netherlands National Trial Register,PACTR - Pan African Clinical Trial Registry, REPEC - Peruvian Clinical Trial Registry,SLCTR - Sri Lanka Clinical Trials Registry, medRxiv Preprints, bioRxiv Preprints, SSRN Preprints, WHO COVID-19 database. 
The last version of the methods, the total number of sources screened, and a living flow diagram and report of the project is updated regularly on our website [17].

The searches will cover from the inception date of each database until the day before submission. No study design, publication status or language restriction will be applied.

The following strategy will be used to identify in the repository the articles relevant for this review:

((coronavir* OR coronovirus* OR betacoronavir* OR "beta-coronavirus" OR "beta-coronaviruses" OR "corona virus" OR "virus corona" OR "corono virus" OR "virus corono" OR hcov* OR "covid-19" OR covid19* OR "covid 19" OR "2019-ncov" OR cv19* OR "cv-19" OR "cv 19" OR "n-cov" OR ncov* OR (wuhan* and (virus OR viruses OR viral)) OR sars* OR sari OR (covid* and (virus OR viruses OR viral)) OR "severe acute respiratory syndrome" OR mers* OR "middle east respiratory syndrome" OR "middle-east respiratory syndrome" OR "covid-19-related" OR "2019-ncov-related" OR "cv-19-related" OR "n-cov-related")) AND ((remdesivir* OR "GS-5734" OR "GS 5734" OR GS5734*))

Other sources

In order to identify articles that might have been missed in the electronic searches, we will do the following:

Screen the reference lists of other systematic reviews and evaluate in full text all the articles they include.

Scan the reference lists of selected guidelines, narrative reviews and other documents.

Review the reference list of each included study. 


\section{Eligibility criteria}

Types of studies

This living review will only include randomised trials.

We will exclude studies evaluating the effects on animal models or in vitro conditions.

Types of participants

We will include trials assessing participants with COVID-19, as defined by the authors of the trials.

Type of interventions

The intervention of interest is remdesivir. We will not restrict our criteria to any dosage, duration, timing or route of administration.

The comparison of interest will be placebo (remdesivir plus optimal treatment versus placebo plus optimal treatment) or no treatment (remdesivir plus optimal treatment versus optimal treatment).

Studies assessing remdesivir plus other drugs will be eligible if the cointerventions are identical in both intervention and comparison groups.

Studies evaluating remdesivir in combination with other active drugs versus placebo or no treatment will also be included.

Type of outcomes

We will not use the outcomes as an inclusion criteria during the selection process. Any article meeting all the criteria except for the outcome criterion will be preliminarily included and assessed in full text.

Our primary outcome of interest will be all-cause mortality at longest follow-up. Secondary outcomes will be invasive mechanical ventilation and adverse effects leading to discontinuation.

We will also extract information on the following outcomes: time to viral clearance, length of hospital stay, extracorporeal membrane oxygenation and serious adverse effects. 


\section{Selection of studies}

The results of the literature search in the repository will be automatically incorporated into the L.OVE platform (automated retrieval), where the titles and abstracts will be independently screened by at least two reviewers against the inclusion criteria. We will obtain the full reports for all records that appear to meet the inclusion criteria or require further analysis to decide about their inclusion.

We will record the reasons for excluding trials in any stage of the search and outline the study selection process in a PRISMA flow diagram adapted for the purpose of this project.

\section{Extraction and management of data}

Using standardised forms, two reviewers will independently extract data from each included study. We will collect the following information: study design, setting, participant characteristics (including disease severity and age) and study eligibility criteria; details about the administered intervention and comparison, including dose and therapeutic scheme, duration, timing (i.e. time after diagnosis) and route of administration; the outcomes assessed and the time they were measured; the source of funding of the study and the conflicts of interest disclosed by the investigators; the risk of bias assessment for each individual study.

We will resolve disagreements by discussion, and one arbiter will adjudicate unresolved disagreements.

\section{Risk of bias assessment}

The risk of bias for each randomised trial will be assessed using the 'risk of bias' tool (RoB 2.0: a revised tool to assess risk of bias in randomised trials) [18]. We will consider the effect of assignment to the intervention for this review. Two reviewers will independently assess five domains of bias for each outcome result of all reported outcomes and time points. These five domains are: bias due to (1) the randomisation process, (2) deviations from intended interventions (effects of assignment to interventions at baseline), (3) missing outcome data, (4) measurement of the outcome, and (5) selection of reported results. Answers to signalling questions and collectively supporting information will lead to a domain-level judgement in the form of 'Low risk of bias', 'Some concerns', or 'High risk of bias'. These domain-level judgements will inform an overall 'risk of bias' judgement for each result. Discrepancies between review authors will be resolved by discussion to reach consensus. If necessary, a third review author will be consulted to achieve a decision.

\section{Measures of treatment effect}

For dichotomous outcomes, we will express the estimate of treatment effect of an intervention as risk ratios along with $95 \%$ confidence intervals $(\mathrm{CI})$.

For continuous outcomes, we will use the mean difference and standard deviation to summarise the data along with $95 \% \mathrm{Cl}$. If continuous outcomes are reported using different scales, the treatment effect will be expressed as a standardised mean difference with $95 \% \mathrm{Cl}$. 


\section{Strategy for data synthesis}

If we include more than one trial we will conduct a formal quantitative synthesis (meta-analysis) for studies clinically homogeneous using RevMan 5 [19], using the inverse variance method with the random-effects model. For any outcomes where data were insufficient to calculate an effect estimate, a narrative synthesis will be presented, describing the direction and size of the effects, along with any reported measure of precision.

\section{Subgroup and sensitivity analysis}

We will perform subgroup analysis according to the definition of severe COVID-19 infection (i.e respiratory failure vs respiratory distress syndrome vs ICU requirement). In case we identify significant differences between subgroups (test for interaction $<0.05$ ) we will report the results of individual subgroups separately.

We will perform sensitivity analysis excluding high risk of bias studies. In cases where the primary analysis effect estimates and the sensitivity analysis effect estimates significantly differ we will either present the low risk of bias (adjusted sensitivity analysis estimates) or present the primary analysis estimates but downgrading the certainty of the evidence because of risk of bias. We will also perform sensitivity analysis if we find substantial clinical heterogeneity on how the condition was defined.

\section{Assessment of certainty of evidence}

The certainty of the evidence for all outcomes will be judged using the Grading of Recommendations Assessment, Development and Evaluation working group methodology (GRADE Working Group) [20], across the domains of risk of bias, consistency, directness, precision and reporting bias. Certainty will be adjudicated as high, moderate, low or very low. For the main comparisons and outcomes, we will prepare Summary of Findings (SoF) tables [21], [22] and also interactive Summary of Findings tables (http://isof.epistemonikos.org/).

\section{Living evidence synthesis}

An artificial intelligence algorithm deployed in the Special L.OVE of Coronavirus (COVID-19)and will provide instant notification of articles with a high likelihood to be eligible. The authors will review them, will decide upon inclusion, and will update the living web version of the review accordingly. We will consider resubmission to a journal if there is a change in the direction of the effect on the critical outcomes or a substantial modification to the certainty of the evidence.

This review is part of a larger project set up to produce multiple parallel systematic reviews relevant to COVID-19 [15]. 


\section{NOTES}

\section{Acknowledgements}

The members of the COVID-19 L.OVE Working Group and Epistemonikos Foundation have made possible to build the systems and compile the information needed by this project. Epistemonikos is a collaborative effort, based on the ongoing volunteer work of over a thousand contributors since 2012.

\section{Roles and contributions}

All the review authors drafted and revised the protocol, conducted article screening and data collection, and drafted and revised the review.

The COVID-19 L.OVE Working Group was created by Epistemonikos and a number of expert teams in order to provide decision makers with the best evidence related to COVID-19. Up-to-date information about the group and its member organisations is available here: epistemonikos.cl/working-group

\section{Competing interests}

All authors declare no financial relationships with any organisation that might have a real or perceived interest in this work. There are no other relationships or activities that might have influenced the submitted work.

\section{Funding}

This project was not commissioned by any organisation and did not receive external funding.

Epistemonikos Foundation is providing training, support and tools at no cost for all the members of the COVID-19 L.OVE Working Group.

\section{PROSPERO registration}

\section{CRD42020183384}

\section{Ethics}

As researchers will not access information that could lead to the identification of an individual participant, obtaining ethical approval was waived.

\section{Data sharing}

All data related to the project will be available. Epistemonikos Foundation will grant access to data. 


\section{REFERENCES}

1. World Health Organization. Director-General's remarks at the media briefing on 2019-nCoV on 11 February 2020. [Internet] World Health Organization; 2020 [Accessed 2020 April 12] Available from: https://www.who.int/dg/speeches/detail/who-director-general-s-remarks-at-the-me dia-briefing-on-2019-ncov-on-11-february-2020 .

2. Hui DS, I Azhar E, Madani TA, et al. The continuing 2019-nCoV epidemic threat of novel coronaviruses to global health - The latest 2019 novel coronavirus outbreak in Wuhan, China. Int $J$ Infect Dis. 2020 Feb;91:264-266. Available from: doi:10.1016/j.jijid.2020.01.009

3. Dong $E$, Du H, Gardner L. An interactive web-based dashboard to track COVID-19 in real time. Lancet Infect Dis. 2020 Feb 19

4. Guan WJ, Ni ZY, Hu Y, et al. Clinical Characteristics of Coronavirus Disease 2019 in China. N Engl J Med 2020. Available from: doi:10.1056/NEJMoa2002032

5. Tavakoli A, Vahdat K, Keshavarz M. Novel Coronavirus Disease 2019 (COVID-19): An Emerging Infectious Disease in the 21st Century. BPUMS. 2020;22(6):432-450. Available from: doi:10.29252/ismj.22.6.432

6. Li LQ, Huang T, Wang YQ, Wang ZP, Liang Y, Huang TB, et al. 2019 novel coronavirus patients' clinical characteristics, discharge rate and fatality rate of meta-analysis. Journal of medical virology. 2020. Available from: doi:10.1002/jmv.25757

7. Global Covid-19 Case Fatality Rates [Internet] UK: Centre for Evidence-Based Medicine [Accessed 2020 April 12]. Available from: https://www.cebm.net/covid-19/global-covid-19-case-fatality-rates/

8. Rodriguez-Morales AJ, Cardona-Ospina JA, Gutiérrez-Ocampo E, et al. Clinical, laboratory and imaging features of COVID-19: A systematic review and meta-analysis. Travel medicine and infectious disease. 2020;101623. Available from: doi:10.1016/j.tmaid.2020.101623

9. Siegel D, Hui HC, Doerffler E, Clarke MO, Chun K, Zhang L, Neville S, Carra E, Lew W, Ross B, Wang Q, Wolfe L, Jordan R, Soloveva V, Knox J, Perry J, Perron M, Stray KM, Barauskas O, Feng JY, Xu Y, Lee G, Rheingold AL, Ray AS, Bannister R, Strickley R, Swaminathan S, Lee WA, Bavari S, Cihlar T, Lo MK, Warren TK, Mackman RL. Discovery and Synthesis of a Phosphoramidate Prodrug of a Pyrrolo[2,1-f][triazin-4-amino] Adenine C-Nucleoside (GS-5734) for the Treatment of Ebola and Emerging Viruses. Journal of medicinal chemistry. 2017;60(5):1648-1661

10. Sheahan TP, Sims AC, Graham RL, Menachery VD, Gralinski LE, Case JB, Leist SR, Pyrc K, Feng JY, Trantcheva I, Bannister R, Park Y, Babusis D, Clarke MO, Mackman RL, Spahn JE, Palmiotti CA, Siegel D, Ray AS, Cihlar T, Jordan R, Denison MR, Baric RS. Broad-spectrum antiviral GS-5734 inhibits both epidemic and zoonotic coronaviruses. Science translational medicine. 2017;9(396).

11. Brandi Williamson, Friederike Feldmann, Benjamin Schwarz, Kimberly Meade-White, Danielle Porter, Jonathan Schulz, Neeltje van Doremalen, Ian Leighton, Claude Kwe 
Yinda, Lizzette Perez-Perez, Atsushi Okumura, Jamie Lovaglio, Patrick Hanley, Greg Saturday, Catharine Bosio, Sarah Anzick, Kent Barbian, Tomas Chilar, Craig Martens, Dana Scott, Vincent Munster, Emmie de Wit. Clinical benefit of remdesivir in rhesus macaques infected with SARS-CoV-2. bioRxiv. 2020

12. U.S. Food and Drug Administration. Coronavirus (COVID-19) Update: FDA Issues Emergency Use Authorization for Potential COVID-19 Treatment. FDA NEWS RELEASE. 2020;

13. Herper M. Inside the NIH's controversial decision to stop its big remdesivir study. STAT. 2020

14. Moher D, Liberati A, Tetzlaff J, Altman DG; PRISMA Group. Preferred reporting items for systematic reviews and meta-analyses: the PRISMA statement. J Clin Epidemiol. 2009;62(10):1006-1012. doi:10.1016/j.jclinepi.2009.06.005

15. Rada G, Verdugo-Paiva F, Ávila C, Morel-Marambio M, Bravo-Jeria R, Pesce F, et al; COVID-19 L.OVE Working Group. Evidence synthesis relevant to COVID-19: a protocol for multiple systematic reviews and overviews of systematic reviews. Medwave 2020;20(3):e7867. Available from: doi:10.5867/medwave.2020.03.7867

16. Github repository [Internet] [Accessed 2020 April 3 Available from: https://github.com/dperezrada/keywords2vec

17. Methods for the special L.OVE of Coronavirus infection [Internet] Santiago: Epistemonikos Foundation [Accessed 2020 April 3]. Available from: https://app.iloveevidence.com/covid-19

18. Xinyao Jin, Bo Pang, Junhua Zhang, et al. Core Outcome Set for Clinical Trials on Coronavirus Disease 2019 (COS-COVID), Engineering, 2020. Available from: doi:10.1016/j.eng.2020.03.002

19. COVID-19 Core Outcomes [Internet]. [Accessed 2020 April 3]. Available from: https://www.covid-19-cos.org/

20. Sterne JAC, Savović J, Page MJ, Elbers RG, Blencowe NS, Boutron I, et al. RoB 2: a revised tool for assessing risk of bias in randomised trials. BMJ. 2019 Aug 28;366:14898. Available from: doi:10.1136/bmj.14898

21. Guyatt GH, Thorlund K, Oxman AD, et al. GRADE guidelines: 13. Preparing summary of findings tables and evidence profiles-continuous outcomes. J Clin Epidemiol 2013 Feb;66(2):173-83. Available from: doi:10.1016/j.jclinepi.2012.08.001

22. Review Manager (RevMan) [Software]. Version 5.3.5 Copenhagen: The Nordic Cochrane Centre, The Cochrane Collaboration, 2014.

23. Guyatt GH, Oxman AD, Vist GE, Kunz R, Falck-Ytter Y, Alonso-Coello P, et al; GRADE Working Group. GRADE: an emerging consensus on rating quality of evidence and strength of recommendations. BMJ. 2008 Apr 26;336(7650):924-6. Available from: doi:10.1136/bmj.39489.470347.AD Available from: doi:10.1016/j.jclinepi.2012.01.012

24. Guyatt GH, Oxman AD, Santesso N, et al. GRADE guidelines: 12. Preparing summary of findings tables-binary outcomes. J Clin Epidemiol [Internet] 2013 Feb [Accessed March 26];66(2):158-72. Available from: doi:10.1016/j.jclinepi.2012.01.012 
